\title{
MULTIGENERATOR GABOR FRAMES ON LOCAL FIELDS
}

\author{
Owais Ahmad and Neyaz A.Sheikh
}

\begin{abstract}
The main objective of this paper is to provide a complete characterization of multigenerator Gabor frames on a periodic set $\Omega$ in $K$. In particular, we provide some necessary and sufficient conditions for the multigenerator Gabor system to be a frame for $L^{2}(\Omega)$. Furthermore, we establish a complete characterization of multigenerator Parseval Gabor frames.

Keywords: Multigenerator Gabor frames, periodic set, signal processing.
\end{abstract}

\section{Introduction}

The concept of frames in a Hilbert space was originally introduced by Duffin and Schaeffer [3] in the context of non-harmonic Fourier series. In signal processing, this concept has become very useful in analyzing the completeness and stability of linear discrete signal representations. Frames did not seem to generate much interest until the ground-breaking work of Daubechies et al. [4]. They combined the theory of continuous wavelet transforms with the theory of frames to introduce wavelet (affine) frames for $L^{2}(\mathbb{R})$. Since then the theory of frames began to be more widely investigated, and now it is found to be useful in signal processing, image processing, harmonic analysis, sampling theory, data transmission with erasures, quantum computing and medicine. Recently, more applications of the theory of frames are found in diverse areas including optics, filter banks, signal detection and in the study of Bosev spaces and Banach spaces. We refer the reader to [1], [5] for an introduction to frame theory and its applications.

The most important concrete realization of frame is Gabor frame. Gabor systems are collections of functions

$$
\mathcal{G}(a, b, \psi)=\left\{M_{m b} T_{n a} \psi(x)=: e^{2 \pi i m a x} \psi(x-n a): m, n \in \mathbb{Z}\right\}
$$

which are built by the combined action of modulations and translations of a single function and hence can be viewed as the set of time-frequency shifts of $\psi(x) \in L^{2}(\mathbb{R})$

Received July 19, 2017; accepted October 19, 2017

2010 Mathematics Subject Classification. Primary 42C15; Secondary 42C40, 43A70 
along the lattice $a \mathbb{Z} \times b \mathbb{Z}$ in $\mathbb{R}^{2}$. Such systems, also called Weyl-Heisenberg systems, were introduced by Gabor [2] with the aim of constructing efficient, time-frequency localized expansions of signals as an infinite linear combinations of elements in [1.1]. The system $\mathcal{G}(a, b, \psi)$ given by [1.1] is called a Gabor frame if there exist constants $A, B>0$ such that

$$
A\|f\|_{2}^{2} \leqslant \sum_{m \in \mathbb{Z}} \sum_{n \in \mathbb{Z}}\left|\left\langle f, M_{m b} T_{n a} \psi\right\rangle\right|^{2} \leqslant B\|f\|_{2}^{2},
$$

holds for every $f \in L^{2}(\mathbb{R})$, and we call the optimal constants $A$ and $B$ the lower frame bound and the upper frame bound, respectively. A tight Gabor frame refers to the case when $A=B$, and a normalized tight frame refers to the case when $A=B=1$. Gabor systems that form frames for $L^{2}(\mathbb{R})$ have a wide variety of applications. One of the most important problem in practice is therefore to determine conditions for Gabor systems to be frames. In practice, once the window function has been chosen, the first question to investigate for Gabor analysis is to find the values of the time-frequency parameters $a, b$ such that $\mathcal{G}(a, b, \psi)$ is a frame. Therefore, the product $a b$ will decide whether the system $\mathcal{G}(a, b, \psi)$ constitutes a frame or is even complete for $L^{2}(\mathbb{R})$ or not. In this context, a useful tool is the Ron and Shen [8] criterion. By using this criterion, Gröchenig et al.[6] have proved that the system $\mathcal{G}(a, b, \psi)$ cannot be a frame for $L^{2}(\mathbb{R})$ if $|a b|>1$ and have also shown that the system $\mathcal{G}(a, b, \psi)$ will form an orthonormal basis for $L^{2}(\mathbb{R})$ if $|a b|=1$.

Gabor analysis is a pervasive signal processing method for decomposing and reconstructing signals from their time frequency projections and also in the context of speech processing, texture segmentation, pattern and object recognition. In order to analyze the dynamic time frequency samples of the signals that contain a wide range of spatial and frequency components, the resolution of which is normally very poor, the single windowed Gabor expansion is not suitable. To address this issue, one of the best choices is the multigenerator Gabor system with a set of multiple windows of various time frequency localizations in frame system. The representation of signals of multiple and time-varying frequencies would have their corresponding windowing templates and resolutions relate to. The concept of multigenerator Gabor system is introduced by Zibulski and Zeevi [12] and they [13] discussed the frame operator associated with the multigenerator Gabor frame by invoking the concept of piecewise Zak transform. They pointed out that the Ballian-Low theorem for the multigenerator Gabor frame is more generalized to the consideration of a scheme of multigenerator which makes it possible to overcome in a way the constraint imposed by the single window in the original theorem. Since then a lot of research [13]-[18] has been carried out in both theory and application aspects of the multigenerator Gabor frame as they can increase the degree of freedom by incorporating windows of various types and widths. For more information on this topic, we refer the reader to [1], [5].

For modeling a signal that appears periodically but intermittently, $a \mathbb{Z}$-periodic set in $\mathbb{R}$ can be used. In this direction, some authors considered the Gabor analysis in $L^{2}(\mathbb{S})$, where $\mathbb{S}$ is an $a \mathbb{Z}$-periodic set in $\mathbb{R}$. Although the classical Gabor analysis 
tools in $L^{2}(\mathbb{S})$ can be adjusted to treat such a scenario by padding with zeros outside the set $\mathbb{S}$, Gabor systems that fit exactly such a scenario might have been more efficient. Gabardo and $\mathrm{Li}[19]$ obtained density results for Gabor systems associated with periodic subsets of the real line. Lian and $\mathrm{Li}[20]$ studied the Gabor frame sets for subspaces. They pointed out that only a periodic $\mathbb{S}$ in $\mathbb{R}$ is suitable for Gabor analysis.

A field $K$ equipped with a topology is called a local field if both the additive and multiplicative groups of $K$ are locally compact Abelian groups. For example, any field endowed with a discrete topology is a local field. For this reason we consider only non-discrete fields. Local fields are essentially of two types (excluding connected local fields $\mathbb{R}$ and $\mathbb{C}$ ). Local fields of the characteristic zero include the $p$-adic field $\mathbb{Q}_{p}$. Examples of local fields of positive characteristic are the Cantor dyadic group and the Vilenkin $p$-groups. Local fields have attracted the attention of several mathematicians, and have found innumerable applications not only in the number theory, but also in the representation theory, division algebras, quadratic forms and algebraic geometry. As a result, local fields are now consolidated as a part of the standard repertoire of contemporary mathematics. For more details we refer the reader to the book by Taibleson [11].

The local field $K$ is a natural model for the structure of Gabor frame systems, as well as a domain upon which one can construct Gabor basis functions. Recently, there has been a substantial body of work concerned with the construction of Gabor frames on $K$ or, more generally, on local fields of positive characteristic. Jiang et al.[7] constructed Gabor frames on local fields of positive characteristic using basic concepts of operator theory and have established a necessary and sufficient conditions for the system $\left\{M_{u(m) b} T_{u(n) a} \psi=: \chi_{m}(b x) \psi(x-u(n) a)\right\}_{m, n \in \mathbb{N}_{0}}$ to be a frame for $L^{2}(K)$. Shah [9] established a complete characterization of Gabor frames on local fields by virtue of two basic equations in the frequency domain and provides the algorithm for constructing an orthonormal Gabor basis for $L^{2}(K)$. Recent results related to Gabor frames on local fields of positive characteristic can be found in $[9],[10]$, and the references therein.

Motivated and inspired by the above work, our aim is to investigate multigenerator Gabor systems on a periodic set in local field and provide complete characterizations for such systems to be frameS. Moreover, necessary and sufficient condition for such a system to be a Parseval Gabor frame. Our results also hold for the Cantor dyadic group and the Vilenkin groups as they are local fields of positive characteristic.

The rest of this paper is organized as follows. In Section 2., we discuss some preliminary facts about Fourier analysis on local fields of positive characteristic and also some results to be used throughout the paper. In Section 3., we establish necessary and sufficient conditions for the multigenerator Gabor system to be a frame for $L^{2}(\Omega)$. In Section 4., we obtain a complete characterization of multigenerator Parseval Gabor frames. 


\section{Preliminaries on local fields and basic facts about frames}

Let $K$ be a field and a topological space. Then $K$ is called a local field if both $K^{+}$ and $K^{*}$ are locally compact Abelian groups, where $K^{+}$and $K^{*}$ denote additive and multiplicative groups of $K$, respectively. If $K$ is any field and is endowed with a discrete topology, then $K$ is a local field. Further, if $K$ is connected, then $K$ is either $\mathbb{R}$ or $\mathbb{C}$. If $K$ is not connected, then it is totally disconnected. Hence by a local field we mean a field $K$ which is locally compact, non-discrete and totally disconnected. $p$-adic fields are examples of local fields. More details can be found in $[11,13]$. In the rest of this paper, we use the symbols $\mathbb{N}, \mathbb{N}_{0}$ and $\mathbb{Z}$ to denote sets of natural, non-negative integers and integers, respectively.

Let $K$ be a local field. Let $d x$ be the Haar measure on the locally compact Abelian group $K^{+}$. If $\alpha \in K$ and $\alpha \neq 0$, then $d(\alpha x)$ is also a Haar measure. Let $d(\alpha x)=|\alpha| d x$. We call $|\alpha|$ the absolute value of $\alpha$. Moreover, the map $x \rightarrow|x|$ has the following properties: (a) $|x|=0$ if and only if $x=0$; (b) $|x y|=|x||y|$ for all $x, y \in K$; and (c) $|x+y| \leqslant \max \{|x|,|y|\}$ for all $x, y \in K$. The property (c) is called the ultrametric inequality. The set $\mathfrak{D}=\{x \in K:|x| \leqslant 1\}$ is called the ring of integers in $K$. Define $\mathfrak{B}=\{x \in K:|x|<1\}$. The set $\mathfrak{B}$ is called the prime ideal in $K$. The prime ideal in $K$ is the unique maximal ideal in $\mathfrak{D}$ and hence as a result $\mathfrak{B}$ is both principal and prime. Since the local field $K$ is totally disconnected, there exists an element of $\mathfrak{B}$ of maximal absolute value. Let $\mathfrak{p}$ be a fixed element of maximum absolute value in $\mathfrak{B}$. Such an element is called the prime element of $K$. Therefore, for such an ideal $\mathfrak{B}$ in $\mathfrak{D}$, we have $\mathfrak{B}=\langle\mathfrak{p}\rangle=\mathfrak{p} \mathfrak{D}$. As it was proved in [11], the set $\mathfrak{D}$ is compact and open. Hence, $\mathfrak{B}$ is compact and open. Therefore, the residue space $\mathfrak{D} / \mathfrak{B}$ is isomorphic to a finite field $G F(q)$, where $q=p^{k}$ for some prime $p$ and $k \in \mathbb{N}$.

Let $\mathfrak{D}^{*}=\mathfrak{D} \backslash \mathfrak{B}=\{x \in K:|x|=1\}$. Then, it can be proved that $\mathfrak{D}^{*}$ is a group of units in $K^{*}$ and if $x \neq 0$, then we may write $x=\mathfrak{p}^{k} x^{\prime}, x^{\prime} \in \mathfrak{D}^{*}$. For the proof of this fact we refer the reader to [11]. Moreover, each $\mathfrak{B}^{k}=\mathfrak{p}^{k} \mathfrak{D}=\left\{x \in K:|x|<q^{-k}\right\}$ is a compact subgroup of $K^{+}$and usually known as the fractional ideals of $K^{+}$. Let $\mathcal{U}=\left\{a_{i}\right\}_{i=0}^{q-1}$ be any fixed full set of coset representatives of $\mathfrak{B}$ in $\mathfrak{D}$, then every element $x \in K$ can be expressed uniquely as $x=\sum_{\ell=k}^{\infty} c_{\ell} \mathfrak{p}^{\ell}$ with $c_{\ell} \in \mathcal{U}$. Let $\chi$ be a fixed character on $K^{+}$that is trivial on $\mathfrak{D}$ but is non-trivial on $\mathfrak{B}^{-1}$. Therefore, $\chi$ is constant on cosets of $\mathfrak{D}$ so if $y \in \mathfrak{B}^{k}$, then $\chi_{y}(x)=\chi(y x), x \in K$. Suppose that $\chi_{u}$ is any character on $K^{+}$, then clearly the restriction $\chi_{u} \mid \mathfrak{D}$ is also a character on $\mathfrak{D}$. Therefore, if $\left\{u(n): n \in \mathbb{N}_{0}\right\}$ is a complete list of the distinct coset representative of $\mathfrak{D}$ in $K^{+}$, then, as it was proved in [13], the set $\left\{\chi_{u(n)}: n \in \mathbb{N}_{0}\right\}$ of distinct characters on $\mathfrak{D}$ is a complete orthonormal system on $\mathfrak{D}$.

The Fourier transform $\hat{f}$ of a function $f \in L^{1}(K) \cap L^{2}(K)$ is defined by

$$
\hat{f}(\xi)=\int_{K} f(x) \overline{\chi_{\xi}(x)} d x .
$$

It is noted that

$$
\hat{f}(\xi)=\int_{K} f(x) \overline{\chi_{\xi}(x)} d x=\int_{K} f(x) \chi(-\xi x) d x .
$$


Furthermore, the properties of the Fourier transform on a local field $K$ are much similar to those on the real line. In particular, the Fourier transform is unitary on $L^{2}(K)$.

We now impose a natural order on the sequence $\{u(n)\}_{n=0}^{\infty}$. We have $\mathfrak{D} / \mathfrak{B} \cong$ $G F(q)$ where $G F(q)$ is a $c$-dimensional vector space over the field $G F(p)$. We choose a set $\left\{1=\zeta_{0}, \zeta_{1}, \zeta_{2}, \ldots, \zeta_{c-1}\right\} \subset \mathfrak{D}^{*}$ such that $\operatorname{span}\left\{\zeta_{j}\right\}_{j=0}^{c-1} \cong G F(q)$. For $n \in \mathbb{N}_{0}$ satisfying

$0 \leq n<q, n=a_{0}+a_{1} p+\cdots+a_{c-1} p^{c-1}, \quad 0 \leq a_{k}<p, \quad$ and $k=0,1, \ldots, c-1$,

we define

$$
u(n)=\left(a_{0}+a_{1} \zeta_{1}+\cdots+a_{c-1} \zeta_{c-1}\right) \mathfrak{p}^{-1} .
$$

Also, for

$$
n=b_{0}+b_{1} q+b_{2} q^{2}+\cdots+b_{s} q^{s}, n \in \mathbb{N}_{0}, 0 \leq b_{k}<q, k=0,1,2, \ldots, s,
$$

we set

$$
u(n)=u\left(b_{0}\right)+u\left(b_{1}\right) \mathfrak{p}^{-1}+\cdots+u\left(b_{s}\right) \mathfrak{p}^{-s} .
$$

This defines $u(n)$ for all $n \in \mathbb{N}_{0}$. In general, it is not true that $u(m+n)=$ $u(m)+u(n)$. But, if $r, k \in \mathbb{N}_{0}$ and $0 \leqslant s<q^{k}$, then $u\left(r q^{k}+s\right)=u(r) \mathfrak{p}^{-k}+u(s)$. Further, it is also easy to verify that $u(n)=0$ if and only if $n=0$ and $\{u(\ell)+u(k)$ : $\left.k \in \mathbb{N}_{0}\right\}=\left\{u(k): k \in \mathbb{N}_{0}\right\}$ for a fixed $\ell \in \mathbb{N}_{0}$. Hereafter we use the notation $\chi_{n}=\chi_{u(n)}, n \geqslant 0$.

Let the local field $K$ be of characteristic $p>0$ and $\zeta_{0}, \zeta_{1}, \zeta_{2}, \ldots, \zeta_{c-1}$ be as above. We define the character $\chi$ on $K$ as follows:

$$
\chi\left(\zeta_{\mu} \mathfrak{p}^{-j}\right)= \begin{cases}\exp (2 \pi i / p), & \mu=0 \text { and } j=1, \\ 1, & \mu=1, \ldots, c-1 \text { or } j \neq 1 .\end{cases}
$$

We also denote the test function space on $K$ by $\mathcal{S}$, i.e., each function $f$ in $\mathcal{S}$ is a finite linear combination of functions of the form $\mathbf{1}_{k}(x-h), h \in K, k \in \mathbb{Z}$, where $\mathbf{1}_{k}$ is the characteristic function of $\mathfrak{B}^{k}$. Then, it is clear that $\mathcal{S}$ is dense in $L^{p}(K), 1 \leqslant p<\infty$, and each function in $\mathcal{S}$ is of compact support and so is its Fourier transform.

A measurable set $\Omega$ in a local field $K$ is said to be $a$ - periodic if $\Omega+u(n) a=\Omega$, for every $n \in \mathbb{N}_{0}$. Let $\Omega$ be an $a$-periodic subset of $K$. Then it is clear that $\Omega$ is $a v$-periodic for every $v \in \mathbb{N}$. Denote $\Omega^{0}=G_{a} \cap \Omega$ and

$$
L^{2}(\Omega)=\left\{f \in L^{2}(K): \operatorname{supp}(f) \subset \Omega\right\}
$$

where $G_{a}=\{x \in \Omega:|x| \leqslant|a|\}$. Clearly, it is a Hilbert space with the inner product in $L^{2}(K)$. 
Definition 2.1. Let $a$ and $b$ be any two fixed elements in $K$. For a fixed positive integer $L$, let $\Psi=\left\{\psi_{1}, \psi_{2}, \ldots, \psi_{L}\right\} \subseteq L^{2}(\Omega)$, define the multi-generator Gabor system

$$
\mathcal{G}(a, b, \Psi):=\left\{M_{u(m) b_{\ell}} T_{u(n) a} \psi^{\ell}=: \chi_{m}\left(b_{\ell} x\right) \psi^{\ell}(x-u(n) a): n, m \in \mathbb{N}_{0}, 1 \leqslant \ell \leqslant L\right\},
$$

where $M_{u(m) b_{\ell}} f(x)=\chi_{m}\left(b_{\ell} x\right) f(x)$ and $T_{u(n) a} f(x)=f(x-u(n) a)$ are the modulation and translation operators defined on $L^{2}(K)$, respectively. We call the Gabor system $\mathcal{G}(a, b, \Psi)$ a Gabor frame for $L^{2}(\Omega)$, if there exist constants $C$ and $D$, $0<C \leqslant D<\infty$ such that

$$
C\|f\|_{2}^{2} \leqslant \sum_{\ell=1}^{L} \sum_{m \in \mathbb{N}_{0}} \sum_{n \in \mathbb{N}_{0}}\left|\left\langle f, M_{u(m) b_{\ell}} T_{u(n) a_{\ell}} \psi^{\ell}\right\rangle\right|^{2} \leqslant D\|f\|_{2}^{2}
$$

The following Lemma follows from the frames associated with shift invariant spaces(see [11] or [1]).

Lemma 2.1. Let $\left\{f_{n}\right\}_{n=1}^{\infty}$ be a family of elements in $L^{2}(K)$ and suppose that for $b>0$,

$$
B=\frac{1}{|b|} \sup _{x \in K} \sum_{k \in \mathbb{N}_{0}}\left|\sum_{n \in \mathbb{N}_{0}} f_{n}(x) \overline{f_{n}\left(x-b^{-1} u(k)\right)}\right|<\infty,
$$

then $\left\{M_{u(m) b} f_{n}: m, n \in \mathbb{N}_{0}\right\}$ is Bessel sequences with the upper bound $B$ for $L^{2}(K)$. Furthermore, if

$$
A=\frac{1}{|b|} \inf _{x \in K}\left\{\sum_{n \in \mathbb{N}_{0}}\left|f_{n}(x)\right|^{2}-\sum_{k \in \mathbb{N}}\left|\sum_{n \in \mathbb{N}_{0}} f_{n}(x) \overline{f_{n}\left(x-b^{-1} u(k)\right)}\right|\right\}>0,
$$

then $\left\{M_{u(m) b} f_{n}: m, n \in \mathbb{N}_{0}\right\}$ is a frame with bounds $A$ and $B$.

\section{Necessary and Sufficient Conditions for Multigenerator Gabor System to be frame for $L^{2}(\Omega)$}

In this section, we establish some necessary and sufficient conditions for the multigenerator Gabor system $\mathcal{G}(a, b, \Psi)$ given by $(2.7)$ to be a frame for $L^{2}(\Omega)$. Before we proceed to the main results, we first provide the relationship between the Gabor system in $L^{2}(K)$ and its subspace $L^{2}(\Omega)$.

Theorem 3.1. Let $\Psi=\left\{\psi_{1}, \psi_{2}, \ldots, \psi_{L}\right\} \subseteq L^{2}(\Omega)$ and $a, b>0$, then the following results hold.

(a) If the Gabor system $\mathcal{G}(a, b, \Psi)$ given by (2.7) is a frame for $L^{2}(K)$, then it is a frame for $L^{2}(\Omega)$.

(b) If the Gabor system $\mathcal{G}(a, b, \Psi)$ given by (2.7) is a Bessel sequence for $L^{2}(\Omega)$ with the upper bound $B$, then it is a Bessel sequence for $L^{2}(K)$ with the same upper bound. 
Proof. The part (a) clearly follows from the fact that $L^{2}(\Omega) \subset L^{2}(K)$. Now we proceed to prove part(b). Suppose that the multigenerator Gabor system $\mathcal{G}(a, b, \Psi)$ given by $(2.7)$ is a Bessel sequence for $L^{2}(\Omega)$. Then there exists a constant $B>0$ such that

$$
\sum_{\ell=1}^{L} \sum_{n \in \mathbb{N}_{0}}\left|\left\langle f, M_{u(m) b} T_{u(n) a} \psi^{\ell}\right\rangle\right|^{2} \leqslant B\|f\|^{\prime} \cdot \quad \forall f \in L^{2}(\Omega) .
$$

Further we observe that

$$
\begin{aligned}
\left\langle f, M_{u(m) b} T_{u(n) a} \psi^{\ell}\right\rangle & =\int_{K} f(x) \overline{\psi^{\ell}(x-u(n) a)} \chi_{m}(b x) d x \\
& =\int_{\Omega} f(x) \overline{\psi^{\ell}(x-u(n) a)} \chi_{m}(b x) d x,
\end{aligned}
$$

as $f \overline{\psi^{\ell}} \in L^{2}(\Omega), 1 \leqslant \ell \leqslant L$, for all $f \in L^{2}(K)$. Therefore, it follows that

$$
\left\langle f, M_{u(m) b} T_{u(n) a} \psi^{\ell}\right\rangle=\left\langle f \mathbf{1}_{\Omega}, M_{u(m) b} T_{u(n) a} \psi^{\ell}\right\rangle
$$

Thus for all $f \in L^{2}(K)$, we have

$$
\begin{aligned}
\sum_{\ell=1}^{L} \sum_{n \in \mathbb{N}_{0}}\left|\left\langle f, M_{u(m) b} T_{u(n) a} \psi^{\ell}\right\rangle\right|^{2} & =\sum_{\ell=1}^{L} \sum_{n \in \mathbb{N}_{0}}\left|\left\langle f \mathbf{1}_{\Omega}, M_{u(m) b} T_{u(n) a} \psi^{\ell}\right\rangle\right|^{2} \\
& \leqslant B\left\|f \mathbf{1}_{\Omega}\right\|^{2} \\
& \leqslant B\|f\|^{2} .
\end{aligned}
$$

This clearly implies that the multigenerator Gabor system $\mathcal{G}(a, b, \Psi)$ given by $(2.7)$ is a Bessel sequence for $L^{2}(K)$ with the same upper bound $B$.

Now we state the sufficient condition for the multigenerator Gabor system $\mathcal{G}(a, b \Psi)$ given by $(2.7)$ to be a frame for $L^{2}(\Omega)$.

Theorem 3.2. Let $\Psi=\left\{\psi_{1}, \psi_{2}, \ldots, \psi_{L}\right\} \subseteq L^{2}(K)$ and $a, b>0$ suppose that

$$
B=\frac{1}{|b|} \sup _{x \in G_{b}-1} \sum_{k \in \mathbb{N}_{0}}\left|\sum_{\ell=1}^{L} \sum_{n \in \mathbb{N}_{0}} T_{(n) a} \psi^{\ell}(x) \overline{T_{u(n) a} \psi^{\ell}\left(x-b^{-1} u(k)\right)}\right|<\infty,
$$

then the multigenerator Gabor system $\mathcal{G}(a, b, \Psi)$ given by (2.7) is a Bessel sequences with the upper bound $B$ for $L^{2}(\Omega)$. Furthermore, if

$A=\frac{1}{|b|} \inf _{x \in G_{b-1}}\left\{\sum_{n \in \mathbb{N}_{0}}\left|\sum_{\ell=1}^{L} T_{u(n) a} \psi^{\ell}(x)\right|^{2}-\sum_{k \in \mathbb{N}}\left|\sum_{\ell=1}^{L} \sum_{n \in \mathbb{N}_{0}} T_{(n) a} \psi^{\ell}(x) \overline{T_{u(n) a} \psi^{\ell}\left(x-b^{-1} u(k)\right)}\right|\right\}>0$,

then the multigenerator Gabor system $\mathcal{G}(a, b, \Psi)$ given by (2.7) is a frame for $L^{2}(\Omega)$ with bounds $A$ and $B$. 
Proof. Define

$$
H_{1}(x)=\sum_{k \in \mathbb{N}_{0}}\left|\sum_{\ell=1}^{L} \sum_{n \in \mathbb{N}_{0}} T_{(n) a} \psi^{\ell}(x) \overline{T_{u(n) a} \psi^{\ell}\left(x-b^{-1} u(k)\right)}\right| .
$$

$$
H_{2}(x)=\sum_{n \in \mathbb{N}_{0}}\left|\sum_{\ell=1}^{L} T_{u(n) a} \psi^{\ell}(x)\right|^{2}-\sum_{k \in \mathbb{N}}\left|\sum_{\ell=1}^{L} \sum_{n \in \mathbb{N}_{0}} T_{(n) a} \psi^{\ell}(x) \overline{T_{u(n) a} \psi^{\ell}\left(x-b^{-1} u(k)\right)}\right|
$$

Clearly $H_{1}$ and $H_{2}$ are $b^{-1}$-periodic functions. Thus

$$
B=\frac{1}{|b|} \sup _{x \in K} \sum_{k \in \mathbb{N}_{0}}\left|\sum_{\ell=1}^{L} \sum_{n \in \mathbb{N}_{0}} T_{(n) a} \psi^{\ell}(x) \overline{T_{u(n) a} \psi^{\ell}\left(x-b^{-1} u(k)\right)}\right|<\infty,
$$

$$
A=\frac{1}{|b|} \inf _{x \in K}\left\{\sum_{n \in \mathbb{N}_{0}}\left|\sum_{\ell=1}^{L} T_{u(n) a} \psi^{\ell}(x)\right|^{2}-\sum_{k \in \mathbb{N}}\left|\sum_{\ell=1}^{L} \sum_{n \in \mathbb{N}_{0}} T_{(n) a} \psi^{\ell}(x) \overline{T_{u(n) a} \psi^{\ell}\left(x-b^{-1} u(k)\right)}\right|\right\}>0
$$

Define

$$
f_{n}(x)=T_{u(k) a} \psi^{\ell}(x),
$$

where $n=\ell+s u(k), \quad 1 \leqslant \ell \leqslant L$. Then, one obtains from (3.9) and (3.10) that

$$
\begin{gathered}
B=\frac{1}{|b|} \sup _{x \in K} \sum_{k \in \mathbb{N}_{0}}\left|\sum_{n \in \mathbb{N}_{0}} f_{n}(x) \overline{f_{n}\left(x-b^{-1} u(k)\right)}\right|<\infty \\
A=\frac{1}{|b|} \inf _{x \in K}\left\{\sum_{n \in \mathbb{N}_{0}}\left|f_{n}(x)\right|^{2}-\sum_{k \in \mathbb{N}}\left|\sum_{n \in \mathbb{N}_{0}} f_{n}(x) \overline{f_{n}\left(x-b^{-1} u(k)\right)}\right|\right\}>0,
\end{gathered}
$$

respectively. By invoking Lemma 2.1, and the fact $L^{2}(\Omega) \subset L^{2}(K)$, the result follows.

Now we prove the necessary condition for the multigenerator Gabor system $\mathcal{G}(a, b, \Psi)$ given by $(2.7)$ to be a frame for $L^{2}(\Omega)$, which depends on the interplay among the functions $\psi^{1}, \psi^{2}, \ldots, \psi^{L}$ and the parameters $a, b_{1}, \ldots, b_{L}$ and the periodic set $\Omega$.

Theorem 3.3. Let $\Psi=\left\{\psi_{1}, \psi_{2}, \ldots, \psi_{L}\right\} \subseteq L^{2}(\Omega)$, and $a, b_{1}, b_{2}, \ldots, b_{L}>0$. Suppose that the Gabor system $\mathcal{G}(a, b, \Psi)$ given by (2.7) is a multigenerator Gabor frame for $L^{2}(\Omega)$ with bounds $A$ and $B$, then

$$
A \mathbf{1}_{\boldsymbol{\Omega}}(x) \leqslant \sum_{\ell=1}^{L}\left\{\frac{1}{\left|b_{\ell}\right|} \sum_{n \in \mathbb{N}_{0}}\left|\psi^{\ell}(x-u(n) a)\right|^{2}\right\} \leqslant B \mathbf{1}_{\boldsymbol{\Omega}}(x), \quad \text { a.e. } K \text {. }
$$


Proof. We first note that $\Omega$ is an $a$-periodic subset of $K$. Therefore, $\psi^{\ell}(\cdot-u(n) a) \in$ $L^{2}(\Omega)$ for all $n \in \mathbb{N}, 1 \leqslant \ell \leqslant L$. Thus

$$
\sum_{\ell=1}^{L}\left\{\frac{1}{\left|b_{\ell}\right|} \sum_{n \in \mathbb{N}_{0}}\left|\psi^{\ell}(x-u(n) a)\right|^{2}\right\}=0, \text { a.e. } K \backslash \Omega .
$$

We establish the proof by contradiction. Assume that the upper bound condition in (3.14) is not true on $\Omega$. Then there exists a measurable set $\Xi \subset \Omega$ with positive measure such that

$$
\sum_{\ell=1}^{L}\left\{\frac{1}{\left|b_{\ell}\right|} \sum_{n \in \mathbb{N}_{0}}\left|\psi^{\ell}(x-u(n) a)\right|^{2}\right\}>B \text { a.e. on } \Xi \text {. }
$$

We can assume that $\Xi$ is contained in a ball $\Upsilon$ with the diameter of $|b|^{-1}$. Setting

$$
\Xi_{0}=\left\{x \in \Xi: \frac{1}{\left|b_{\ell}\right|} \sum_{n \in \mathbb{N}_{0}}\left|\psi^{\ell}(x-u(n) a)\right|^{2} \geqslant \frac{1}{\left|b_{\ell}\right|}+B\right\}
$$

and

$\Xi_{k}=\left\{x \in \Xi: \frac{1}{\left|b_{\ell}\right|(k+1)}+B \leqslant \frac{1}{\left|b_{\ell}\right|} \sum_{n \in \mathbb{N}_{0}}\left|\psi^{\ell}(x-u(n) a)\right|^{2}<\frac{1}{\left|b_{\ell}\right| k}+B\right\}, \quad k \in \mathbb{N}$.

Thus we obtain a partition of $\Xi$ into disjoint measurable sets among which at least one say, $\Xi_{s}$, has positive measure.

Now consider the function $f=\mathbf{1}_{\Xi_{s}}$, the characteristic function on $\Xi_{s}$ and note that $\|f\|^{2}=\left|\Xi_{s}\right|$. Clearly, for any $n \in \mathbb{N}_{0}$, the function $f \overline{T_{u(n) a} \psi^{\ell}}$ has support in $\Xi_{s}$. Since $\Xi_{s}$ is contained in a ball $\Upsilon$ with the diameter of $|b|^{-1}$ and the functions $\left\{\sqrt{\left|b_{\ell}\right|} \chi_{m}\left(b_{\ell} x\right): m \in \mathbb{N}_{0}, 1 \leqslant \ell \leqslant L\right\}$ constitutes an orthonormal basis for $L^{2}(\Upsilon)$ for every ball $\Upsilon$ of the diameter $|b|^{-1}$, we have

$$
\begin{aligned}
\sum_{m \in \mathbb{N}_{0}}\left|\left\langle f, M_{u(m) b_{\ell}} T_{u(n) a_{\ell}} \psi^{\ell}\right\rangle\right|^{2} & =\sum_{m \in \mathbb{N}_{0}}\left|\left\langle f \overline{T_{u(n) a_{\ell}} \psi^{\ell}}, M_{u(m) b_{\ell}}\right\rangle\right|^{2} \\
& =\frac{1}{\left|b_{\ell}\right|} \int_{K}|f(x)|^{2}\left|\psi^{\ell}(x-u(n) a)\right|^{2} d x .
\end{aligned}
$$

Thus

$$
\begin{aligned}
\sum_{m \in \mathbb{N}_{0}}\left|\left\langle f, M_{u(m) b_{\ell}} T_{u(n) a_{\ell}} \psi^{\ell}\right\rangle\right|^{2} & =\frac{1}{\left|b_{\ell}\right|} \int_{\Xi_{s}}|f(x)|^{2} \sum_{n \in \mathbb{N}_{0}}\left|\psi^{\ell}(x-u(n) a)\right|^{2} d x \\
& \geqslant\left\{B+\frac{1}{\left|b_{\ell}\right|(s+1)}\right\}\|f\|^{2} .
\end{aligned}
$$


This is a contradiction to the assumption that $B$ is the upper frame bound for the multigenerator Gabor system $\mathcal{G}(a, b, \Psi)$ given by $(2.7)$. In a similar vein, we can show that if the lower bound condition in (3.14) is violated, then $A$ cannot be the lower bound for the multigenerator Gabor system $\mathcal{G}(a, b, \Psi)$ given by $(2.7)$.

\section{Characterizations of Parseval Multigenerator Gabor Frame}

In this section, we will provide the characterization of Parseval multigenerator Gabor frames. The following Lemma is very useful in this section.

Lemma 4.1. Let $f$ be a bounded measurable function with compact support and let $\Psi=\left\{\psi_{1}, \psi_{2}, \ldots, \psi_{L}\right\} \subseteq L^{2}(\Omega)$, then for $a, b>0$ and $1 \leqslant \ell \leqslant L$, we have

$$
\begin{aligned}
\sum_{m \in \mathbb{N}_{0}} \sum_{n \in \mathbb{N}_{0}} & \left|\left\langle f, M_{u(m) b} T_{u(n) a} \psi^{\ell}\right\rangle\right|^{2} \\
\left.=\frac{1}{|b|} \int_{K}|f(x)|^{2} \mid \sum_{n \in \mathbb{N}_{0}} \psi^{\ell}(x-u(n) a)\right)\left.\right|^{2} d x & \\
& \quad+\sum_{k \in \mathbb{N}_{0}} \frac{1}{|b|} \int_{K} \overline{f(x)} \psi^{\ell}(x-u(n) a) \\
& \times \sum_{n \in \mathbb{N}} \psi^{\ell}\left(x+b^{-1} u(k)\right) \overline{\psi^{\ell}\left(x+b^{-1} u(k)-u(n) a\right)} d x
\end{aligned}
$$


Proof. We have

$$
\begin{aligned}
\sum_{m \in \mathbb{N}_{0}} \sum_{n \in \mathbb{N}_{0}} & \left|\left\langle f, M_{u(m) b} T_{u(n) a} \psi^{\ell}\right\rangle\right|^{2} \\
& =\sum_{m \in \mathbb{N}_{0}} \sum_{n \in \mathbb{N}_{0}} \int_{K}\left|f(x) \overline{\psi^{\ell}(x-u(n) a)} \overline{\chi_{m}(b x)} d x\right|^{2} \\
& =\sum_{m \in \mathbb{N}_{0}} \sum_{n \in \mathbb{N}_{0}} \int_{K}\left|f(x+u(n) a) \overline{\psi^{\ell}(\xi)} \overline{\chi_{m}(b x)} d x\right|^{2} \\
& =\sum_{n \in \mathbb{N}_{0}} \frac{1}{|b|} \int_{K} \overline{f(x+u(n) a)} \psi^{\ell}(\xi) \\
& =\sum_{n \in \mathbb{N}_{0}} \frac{1}{|b|} \int_{K} \mid \frac{\sum_{\ell \in \mathbb{N}_{0}} f\left(x+b^{-1} u(\ell)+u(n) a\right) \overline{\psi^{\ell}\left(x+b^{-1} u(\ell)\right)}, d x}{\times(n) a)\left.\right|^{2}\left|\psi^{\ell}(x)\right|^{2} d x} \\
& +\sum_{n \in \mathbb{N}_{0}} \frac{1}{|b|} \int_{K} \frac{1}{f(x+u(n) a)} \int_{K \in \mathbb{N}_{0}^{\ell}(x)} \frac{1}{|b|} \int_{K} \frac{\left.|f(x)|^{2} \mid \psi^{\ell}(x-u(n) a)\right)\left.\right|^{2} d x}{f(x)} \psi^{\ell}(x-u(n) a) \\
& \times \sum_{k \in \mathbb{N}} f\left(x+b^{-1} u(k)+u(n) a\right) \overline{\psi^{\ell}\left(x+b^{-1} u(k)\right)} d x
\end{aligned}
$$

Theorem 4.1. Let $\Psi=\left\{\psi_{1}, \psi_{2}, \ldots, \psi_{L}\right\} \subseteq L^{2}(\Omega)$, and $a, b_{1}, b_{2}, \ldots, b_{L}>0$. Suppose that the Gabor system $\mathcal{G}(a, b, \Psi)$ given by (2.7) is a tight frame for $L^{2}(\Omega)$ with $A=1$, then

$$
\left.\left.\sum_{\ell=1}^{L} \frac{1}{\left|b_{\ell}\right|}\left\{\sum_{n \in \mathbb{N}_{0}} \psi^{\ell}(x-u(n) a)\right)\right|^{2}\right\}=\mathbf{1}_{\Omega}, \text { a.e. } K \text {. }
$$


Furthermore, if $b_{1}=b_{2}=\cdots=b_{L}=b($ say $)$, then for $k \in \mathbb{N}$, we have

$$
\left.\left.\sum_{\ell=1}^{L}\left\{\sum_{n \in \mathbb{N}_{0}} \psi^{\ell}(x-u(n) a)\right)\right|^{2}\right\}=|b| \mathbf{1}_{\Omega}, \text { a.e. } K .
$$

$$
\sum_{\ell=1}^{L}\left\{\sum_{k \in \mathbb{N}} \psi^{\ell}\left(x+b^{-1} u(m)\right) \overline{\psi^{\ell}\left(x+b^{-1} u(k)-u(n) a\right)}\right\}=0,
$$

hold a.e in $K$.

Proof. Define

$$
\nu^{1}=\min \left\{\frac{1}{\left|b_{\ell}\right|}: 1 \leqslant \ell \leqslant L\right\}
$$

Consider

$$
\mathcal{D}=\left\{f: f \in L^{2}(\Omega) \text { and supp } f \subset\left(G_{\nu^{1}} \cap \Omega\right)\right\}
$$

Since $\mathcal{G}(a, b, \Psi)$ given by $(2.7)$ is a tight frame for $L^{2}(\Omega)$ with $A=1$. Then

$$
\sum_{\ell=1}^{L} \sum_{m \in \mathbb{N}_{0}} \sum_{n \in \mathbb{N}_{0}}\left|\left\langle f, M_{u(m) b} T_{u(n) a} \psi^{\ell}\right\rangle\right|^{2} \leqslant\|f\|^{2}, \quad \forall f \in \mathcal{D} .
$$

By Invoking Lemma4.1, for all $f \in \mathcal{D}$ and fixed $\ell, n$, we have

$$
\begin{aligned}
\sum_{m \in \mathbb{N}_{0}} \sum_{n \in \mathbb{N}_{0}}\left|\left\langle f, M_{u(m) b} T_{u(n) a} \psi^{\ell}\right\rangle\right|^{2} & =\sum_{m \in \mathbb{N}_{0}}\left|\int_{K} f(x) \overline{\psi^{\ell}(x-u(n) a) \chi_{m}\left(b_{\ell} x\right)} d x\right|^{2} \\
& =\frac{1}{\left|b_{\ell}\right|} \int_{K}\left|f(x) \overline{\psi^{\ell}(x-u(n) a)}\right|^{2} d x \\
& =\frac{1}{\left|b_{\ell}\right|} \int_{G_{\nu^{1}}}\left|f(x) \psi^{\ell}(x-u(n) a)\right|^{2} d x
\end{aligned}
$$

Thus for any $f \in \mathcal{D}$, we have

$$
\left.\int_{G_{\nu^{1}}}|f(x)|^{2} d x=\left.\int_{G_{\nu^{1}}}|f(x)|^{2} \sum_{\ell=1}^{L}\left\{\sum_{n \in \mathbb{N}_{0}} \psi^{\ell}(x-u(n) a)\right)\right|^{2}\right\} d x
$$

implies that

$$
\left.\left.\sum_{\ell=1}^{L}\left\{\sum_{n \in \mathbb{N}_{0}} \psi^{\ell}(x-u(n) a)\right)\right|^{2}\right\}=1 \quad \text { a.e. } G_{\nu^{1}} \cap \Omega .
$$


which gives the desired result (4.1) and its particular case (4.2).

Next we proceed to prove (4.3). For fixed $\ell, 1 \leqslant \ell \leqslant L$, by using Lemma 4.1, we have (4.10)

$$
\begin{aligned}
\sum_{m \in \mathbb{N}_{0}} \sum_{n \in \mathbb{N}_{0}} & \left|\left\langle f, M_{u(m) b} T_{u(n) a} \psi^{\ell}\right\rangle\right|^{2} \\
= & \left.\frac{1}{|b|} \int_{K}|f(x)|^{2} \mid \sum_{n \in \mathbb{N}_{0}} \psi^{\ell}(x-u(n) a)\right)\left.\right|^{2} d x \\
+ & \sum_{k \in \mathbb{N}_{0}} \frac{1}{|b|} \int_{K} \overline{f(x)} \psi^{\ell}(x-u(n) a) \\
& \quad \times \sum_{n \in \mathbb{N}} \psi^{\ell}\left(x+b^{-1} u(k)\right) \overline{\psi^{\ell}\left(x+b^{-1} u(k)-u(n) a\right)} d x
\end{aligned}
$$

Then,

(4.11)

$$
\begin{aligned}
\sum_{\ell=1}^{L} \sum_{m \in \mathbb{N}_{0}} \sum_{n \in \mathbb{N}_{0}} & \left|\left\langle f, M_{u(m) b} T_{u(n) a} \psi^{\ell}\right\rangle\right|^{2} \\
= & \left.\int_{K}|f(x)|^{2} \mid \sum_{\ell=1}^{L} \frac{1}{|b|} \sum_{n \in \mathbb{N}_{0}} \psi^{\ell}(x-u(n) a)\right)\left.\right|^{2} d x \\
+ & \sum_{k \in \mathbb{N}_{0}} \frac{1}{|b|} \int_{K} \overline{f(x)} \psi^{\ell}(x-u(n) a) \\
& \times \sum_{\ell=1}^{L}\left(\sum_{n \in \mathbb{N}} \psi^{\ell}\left(x+b^{-1} u(k)\right) \overline{\psi^{\ell}\left(x+b^{-1} u(k)-u(n) a\right)}\right) d x
\end{aligned}
$$

By combining (4.11) with (4.2), it follows that

$(4.12)$

$$
\begin{aligned}
& \sum_{k \in \mathbb{N}_{0}} \frac{1}{|b|} \int_{K} \overline{f(x)} \psi^{\ell}(x-u(n) a) \\
& \times \sum_{\ell=1}^{L}\left(\sum_{n \in \mathbb{N}} \psi^{\ell}\left(x+b^{-1} u(k)\right) \overline{\psi^{\ell}\left(x+b^{-1} u(k)-u(n) a\right)}\right) d x=0 .
\end{aligned}
$$

By using the change $u(k) \rightarrow-u(k)$, it can be seen that the contribution in the above sum for any value of $u(k)$ is a complex conjugate of the contribution from 
the value $-u(k)$. Therefore, we have

$$
\sum_{k \in \mathbb{N}} R e\left\{\frac{1}{|b|} \int_{K} \overline{f(x)} \psi^{\ell}(x-u(n) a) \Theta_{k}(x) d x\right\}=0 .
$$

where

$$
\Theta_{k}(x)=\sum_{\ell=1}^{L}\left(\sum_{n \in \mathbb{N}} \psi^{\ell}\left(x+b^{-1} u(k)\right) \overline{\psi^{\ell}\left(x+b^{-1} u(k)-u(n) a\right)}\right)=0 .
$$

To establish the required result, we consider three cases. First we consider the case when $x \in \Omega$. Since $\Omega$ is an $a$ - periodic set, then $x-u(n) a \in \Omega$ for all $n \in \mathbb{N}_{0}$. Therefore

$$
\psi^{\ell}(x-u(n) a)=0, \quad \forall n \in \mathbb{N}_{0}
$$

Thus

$$
\sum_{\ell=1}^{L}\left(\sum_{n \in \mathbb{N}} \psi^{\ell}\left(x+b^{-1} u(k)\right) \overline{\psi^{\ell}\left(x+b^{-1} u(k)-u(n) a\right)}\right)=0, \quad \forall k \in \mathbb{N} .
$$

The second case is when $x-b^{-1} u(k) \notin \Omega$ for fixed $k \in \mathbb{N}$. Then $x-u(n) a-b^{-1} u(k) \notin$ $\Omega$ for all $n \in \mathbb{N}_{0}$. Therefore

$$
\sum_{\ell=1}^{L}\left(\sum_{n \in \mathbb{N}} \psi^{\ell}\left(x+b^{-1} u(k)\right) \overline{\psi^{\ell}\left(x+b^{-1} u(k)-u(n) a\right)}\right)=0 .
$$

The third case is when $x \in \Omega$ and $x-b^{-1} u(k) \in \Omega$ for fixed $k \in \mathbb{N}$. Let $\Gamma$ be any ball of the radius at most $b^{-1}$ and denote $\Gamma \cap \Omega$ by $\Gamma^{0}$ and $\left(\Gamma-b^{-1} u(k)\right) \cap\left(\Omega+b^{-1} u(k)\right.$ by $\Gamma^{\prime}$. If the measure of $\Gamma \cap \Gamma^{\prime}$ is zero, then $x \notin \Gamma^{0}$ a.e. or $x \notin \Gamma-b^{-1} u(k)$ a.e, thus

$$
\sum_{\ell=1}^{L}\left(\sum_{n \in \mathbb{N}} \psi^{\ell}\left(x+b^{-1} u(k)\right) \overline{\psi^{\ell}\left(x+b^{-1} u(k)-u(n) a\right)}\right)=0 .
$$

If the measure of $\Gamma^{0} \cap \Gamma^{\prime}$ is positive. We define a function $f \in L^{2}(\Omega)$ by

$$
f(x)=\left\{\begin{aligned}
\exp \left\{-\arg \Theta_{k_{0}}(x)\right\}, & x \in \Gamma^{0} \cap \Gamma^{\prime} \\
1, & x \in \Gamma^{0} \cap \Gamma^{\prime}-b^{-1} u(k), \\
0, & \text { otherwise. }
\end{aligned}\right.
$$


Then, by (4.13) we obtain

$$
\int_{\Gamma^{0} \cap \Gamma^{\prime}}\left|\Theta_{k_{0}}(x)\right| d x=0
$$

It follows that $\Theta_{k_{0}(x)}=0$, a.e, on $\Gamma \cap \Gamma^{\prime}$. Since $\Gamma$ is an arbitrary ball of the radius at most $\frac{1}{b}$, we conclude that $\Theta_{k_{0}}(x)=0$, a.e, in $\Omega$. A simple computation shows that

$$
\Theta_{-k_{0}}(x)=\Theta_{k_{0}}\left(x+b^{-1} u\left(k_{0}\right)\right)
$$

Thus the desired result follows.

To proceed further, we first define some notations. For $b_{1}, b_{2}, \cdots, b_{L}>0$, we define

$$
\nu^{1}=\min _{1 \leqslant \ell \leqslant L}\left\{\frac{1}{\left|b_{\ell}\right|}\right\}
$$

and for $j \geqslant 2$,

$$
\nu^{j}=\min _{1 \leqslant \ell \leqslant L}\left\{\frac{1}{\left|b_{\ell}\right|}:\left|b_{\ell}\right|<\frac{1}{\nu^{j-1}}\right\} .
$$

Also, we define

$$
\mathcal{I}_{j}=\left\{\ell:\left|b_{\ell}\right|=\frac{1}{\nu^{j}}, 1 \leqslant \ell \leqslant L\right\} .
$$

Then there exists a unique $j_{0} \in \mathbb{N}$ such that

$$
\begin{aligned}
& \mathcal{I} \neq \phi \text { for } 1 \leqslant j \leqslant j_{0}, \\
& \mathcal{I}_{j_{1}} \cap \mathcal{I}_{j_{2}}=\phi, \text { for } j_{1} \neq j_{2}, \\
& \bigcup_{j=1}^{j_{0}} \mathcal{I}_{j}=\{1,2, \cdots, L\} .
\end{aligned}
$$

Theorem 4.2. Let $j_{0}$ be a unique positive integer satisfying (4.21). Suppose that $\Psi=\left\{\psi_{1}, \psi_{2}, \ldots, \psi_{L}\right\} \subseteq L^{2}(\Omega)$, and $a, b_{1}, b_{2}, \ldots, b_{L}>0$ satisfy

$$
\left.\left.\sum_{\ell=1}^{L} \frac{1}{\left|b_{\ell}\right|}\left\{\sum_{n \in \mathbb{N}_{0}} \psi^{\ell}(x-u(n) a)\right)\right|^{2}\right\}=\mathbf{1}_{\Omega},
$$

$$
\sum_{\ell \in \mathcal{I}_{j}}\left\{\sum_{m \in \mathbb{N}_{0}} \psi^{\ell}\left(x+b^{-1} u(m)\right) \overline{\psi^{\ell}\left(x+b^{-1} u(k)-u(m) a\right)}\right\}=0, \text { for } k \in \mathbb{N}, 1 \leqslant j \leqslant j_{0}
$$

a.e in $K$. Then the Gabor system $\mathcal{G}(a, b, \Psi)$ given by (2.7) is a tight frame for $L^{2}(\Omega)$ with $A=1$. 
Proof. For fixed $\ell=1,2, \ldots, L$, using Lemma 4.1, we obtain (4.24)

$$
\begin{aligned}
\sum_{m \in \mathbb{N}_{0}} \sum_{n \in \mathbb{N}_{0}} & \left|\left\langle f, M_{u(m) b_{\ell}} T_{u(n) a} \psi^{\ell}\right\rangle\right|^{2} \\
= & \left.\frac{1}{\left|b_{\ell}\right|} \int_{K}|f(x)|^{2} \mid \sum_{n \in \mathbb{N}_{0}} \psi^{\ell}(x-u(n) a)\right)\left.\right|^{2} d x \\
& +\sum_{k \in \mathbb{N}_{0}} \frac{1}{\left|b_{\ell}\right|} \int_{K} \overline{f(x)} \psi^{\ell}(x-u(n) a) \\
& \times\left(\sum_{n \in \mathbb{N}} \psi^{\ell}\left(x+b_{\ell}^{-1} u(k)\right) \overline{\psi^{\ell}\left(x+b_{\ell}^{-1} u(k)-u(n) a\right)}\right) d x
\end{aligned}
$$

which implies that,

$$
\begin{aligned}
\sum_{\ell=1}^{L} \sum_{m \in \mathbb{N}_{0}} \sum_{n \in \mathbb{N}_{0}} & \left|\left\langle f, M_{u(m) b_{\ell}} T_{u(n) a} \psi^{\ell}\right\rangle\right|^{2} \\
& \left.=\int_{K} \sum_{\ell=1}^{L} \frac{1}{\left|b_{\ell}\right|}|f(x)|^{2} \mid \sum_{n \in \mathbb{N}_{0}} \psi^{\ell}(x-u(n) a)\right)\left.\right|^{2} d x+
\end{aligned}
$$

where

(4.26)

$$
\begin{aligned}
(\boldsymbol{\square})=\int_{K} \sum_{\ell=1}^{L} \sum_{k \in \mathbb{N}_{0}} \frac{1}{\left|b_{\ell}\right|} & \overline{f(x)} \psi^{\ell}(x-u(n) a) \\
& \left(\sum_{n \in \mathbb{N}} \psi^{\ell}\left(x+b_{\ell}^{-1} u(k)\right) \overline{\psi^{\ell}\left(x+b_{\ell}^{-1} u(k)-u(n) a\right)}\right) d x .
\end{aligned}
$$

On combining (4.26) with (4.22), it follows that

$$
\sum_{\ell=1}^{L} \sum_{m \in \mathbb{N}_{0}} \sum_{n \in \mathbb{N}_{0}}\left|\left\langle f, M_{u(m) b_{\ell}} T_{u(n) a} \psi^{\ell}\right\rangle\right|^{2}=\int_{K}|f(x)|^{2} d x+(\mathbf{\square}) .
$$

Define

$$
\Theta_{k}^{j}(x)=\sum_{\ell \in \mathcal{I}_{j}}\left\{\sum_{m \in \mathbb{N}_{0}} \psi^{\ell}\left(x+b^{-1} u(m)\right) \overline{\psi^{\ell}\left(x+b^{-1} u(k)-u(m) a\right)}\right\} \text { for } 1 \leqslant j \leqslant j_{0}
$$

Then, from (4.23) we obtain 


$$
(\mathbf{\square})=\sum_{k \in \mathbb{N}}\left\{\int_{K} \sum_{j=1}^{j_{0}} \frac{1}{\left|b_{j}\right|} \overline{f(x)} f\left(x-b_{j}^{-1} u(k) \Theta_{k}^{j}(x)\right\}=0 .\right.
$$

From this together with (4.27), it follows that

$$
\sum_{\ell=1}^{L} \sum_{m \in \mathbb{N}_{0}} \sum_{n \in \mathbb{N}_{0}}\left|\left\langle f, M_{u(m) b_{\ell}} T_{u(n) a} \psi^{\ell}\right\rangle\right|^{2}=\int_{K}|f(x)|^{2} d x=\|f\|, \quad \forall f \in L^{2}(\Omega) .
$$

Therefore, the Gabor system $\mathcal{G}(a, b, \Psi)$ given by $(2.7)$ is a tight frame for $L^{2}(\Omega)$ with $A=1$.

From the above two theorems, we obtain the following theorem, which is a necessary and sufficient condition for the multigenerator Parseval Gabor frame.

Theorem 4.3. Let $\Psi=\left\{\psi_{1}, \psi_{2}, \ldots, \psi_{L}\right\} \subseteq L^{2}(\Omega)$, and $a, b>0$. Then the Gabor system $\mathcal{G}(a, b, \Psi)$ given by (2.7) is a tight frame for $L^{2}(\Omega)$ with $A=1$ if and only if

$$
\begin{gathered}
\left.\left.\sum_{\ell=1}^{L} \frac{1}{|b|}\left\{\sum_{n \in \mathbb{N}_{0}} \psi^{\ell}(x-u(n) a)\right)\right|^{2}\right\}=\mathbf{1}_{\Omega}, \\
\sum_{\ell=1}^{L}\left\{\sum_{n \in \mathbb{N}} \psi^{\ell}\left(x+b^{-1} u(n)\right) \overline{\psi^{\ell}\left(x+b^{-1} u(k)-u(n) a\right)}\right\}=0,
\end{gathered}
$$

hold a.e in $K$.

\section{REF E R E N C ES}

1. O. Christensen, An Introduction to Frames and Riesz Bases, Birkhäuser, Boston, 2015.

2. D. Gabor, Theory of communications, J. Inst. Elect. Engn., 93, 429-457, 1946.

3. R. J. Duffin and A. C. Shatffer, A class of nonharmonic Fourier series, Trans. Amer. Math. Soc. 72 (1952) 341-366.

4. I. Daubechies, A. Grossmann and Y. Meyer, Painless non-orthogonal expansions, J. Math. Phys. 27(5) (1986) 1271-1283.

5. K. Gröchenig, Foundation of Time-Frequency Analysis, Birkhäuser, Boston, 2001.

6. K. Gröchenig, A. J. Janssen, N. Kaiblinger and GE. Pfander, Note on Bsplines, wavelet scaling functions, and Gabor frames, IEEE Trans. Informat. Theory, 49(12), 3318-3320, 2003.

7. D. LI and H. K. JIANG, Basic results of Gabor frame on local fields, Chinese Ann. Math. Series B, 28(2), 165-176, 2007. 
8. A. Ron and Z. Shen, Weyl-Heisenberg frames and Riesz bases in $L^{2}\left(\mathbb{R}^{d}\right)$, Duke Math. J, 89, 237- 282, 1997.

9. F. A. SнAн, A characterization of tight Gabor frame on local fields of positive characteristion, Preprint, 2017.

10. F. A. SнAн, Gabor frames on a half-line, J. Contemp. Math. Anal., 47(5), 251-260, 2012 .

11. M. H. TAibleson, Fourier Analysis on Local Fields, Princeton University Press, Princeton, NJ, 1975.

12. M. Zibulskiand Y. Y. ZeEvi, Discrete multiwindow Gabor-type transforms, IEEE Trans. on Signal Proc., 45 (6), 1428-1442, 1997.

13. M. ZiBUlsKI and Y. Y. ZeEvi, Analysis of multiwindow Gabor-type schemes by frame methods, Appl. and Comput. Harmon. Anal., 4 (2), 188-221, 1997.

14. A. AkAN and L. F. ChAPARRo, Multi-window Gabor expansion for evolutionary spectral analysis, IEEE Trans. on Sig. Proc., 63 (3), 249-262, 1997.

15. S. Li, Discrete multi-Gabor expansions, IEEE Trans. on Informat. Theory, 45 (6) 1954-1967, 1999.

16. S. LI, Proportional nonuniform multi-Gabor expansions, EURASIP J. on Appl, Sig. Proc., 17, 2723-2731, 2004.

17. N. K. Subbanna and Y. Y. ZeEvi, Existence conditions for discrete noncanonical multiwindow Gabor schemes, IEEE Trans. on Signal Proc., 55 (1)0, 5113-5117, 2007.

18. Y. Z. LI and Q. F. LIAN, Multi-window Gabor frames and oblique Gabor duals on discrete periodic sets, Sc. China, 54 (5), 987-1010, 2011.

19. J. P. Gabardo and Y. Z. LI, Density results for Gabor systems associated with periodic subsets of the real line, Jour. of Approx. Th., 157 (2), 172-192, 2009.

20. Q. F. LiAn and Y. Z. Li, Gabor frame sets for subspaces, Adv. in Comput. Math., 34 (4), 391-411, 2011.

21. A. Ron and Z. W. Shen, Frames and stable bases for shift-invariant subspaces of $L^{2}\left(R^{d}\right)$, Canad. Jour. of Math., 47 (5), 1051-1094, 1995.

Owais Ahmad

Department of Mathematics,

National Institute of Technology,

P. O. Box 190006

Jammu and Kashmir, India

siawoahmad@gmail.com

Neyaz Ahmad Sheikh

Department of Mathematics,

National Institute of Technology,

P. O. Box 190006

Jammu and Kashmir, India

neyaznit@yahoo.co.in 